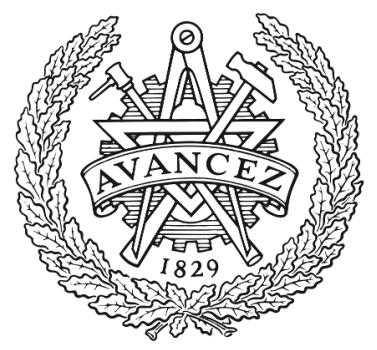

CHALMERS

UNIVERSITY OF TECHNOLOGY

\title{
Tyre Pattern Features and their Effects on Passenger Vehicle Drag
}

Downloaded from: https://research.chalmers.se, 2023-04-26 07:13 UTC

Citation for the original published paper (version of record):

Hobeika, T., Sebben, S. (2018). Tyre Pattern Features and their Effects on Passenger Vehicle Drag. SAE International Journal of Passenger Cars - Mechanical Systems, 11(5): 401-413. http://dx.doi.org/10.4271/2018-01-0710

N.B. When citing this work, cite the original published paper. 


\title{
Tyre Pattern Features and their Effects on Passenger Vehicle Drag
}

\author{
Teddy Hobeika and Simone Sebben \\ Chalmers University of Technology
}

\begin{abstract}
In light of the drive for energy efficiency and low $\mathrm{CO}_{2}$ emissions, extensive research is performed to reduce vehicle aerodynamic drag. The wheels are relatively shielded from the main flow compared to the exterior of the passenger car, however, they are typically responsible for around $25 \%$ of the overall vehicle drag. This contribution is large as the wheels and tyres protrude into the flow and change the flow structure around the vehicle underbody. Given that the tyre is the first part of the wheel to get in contact with the oncoming flow, its shape and features have a significant impact on the flow pattern that develops.
\end{abstract}

This study aims at identifying the general effects of two main tyre features, the longitudinal rain grooves and lateral pattern grooves, using both CFD and wind tunnel tests. This is performed by cutting generic representations of these details into identical slick tyres. Combinations of the two resulted in four physical tyre patterns that are tested on both a production and a closed rim. The test setup is reproduced in CFD taking the tyre deformation under loading into account. Due to the tyre's deformation, Moving Reference Frame - grooves (MRFg) was used to model rotation, while the rim spokes were modelled with the sliding mesh approach.

The results indicate that the rain grooves play a significant role in reducing drag when introduced on a slick tyre both in test and simulations, while the results from adding lateral grooves were less consistent dependent on the rim-tyre combination. The interaction between the longitudinal and lateral grooves could be observed on the overall vehicle drag. In general, CFD is able to predict the drag changes for different tyre patterns with good accuracy for the open rim, however the closed rim case proved to be more challenging.

\section{Introduction}

Active steps are being taken by governments, manufacturers, and even consumers to reduce $\mathrm{CO}_{2}$ emissions in an attempt to contain global warming. This is especially important for road vehicle manufacturers, as road vehicles are responsible for more than $15 \%$ of the total $\mathrm{CO}_{2}$ emissions in the EU, according to the European Energy Agency [1]. This has also led to the introduction of strict regulations with more realistic driving cycles, as well as a thorough procedure for measuring $\mathrm{CO}_{2}$ emissions of road vehicles. This procedure is known as the Worldwide harmonized Light vehicles Test Procedure (WLTP) and is gradually being implemented. The European Union Council also aims to bring $\mathrm{CO}_{2}$ emissions for car fleets down to a $95 \mathrm{~g} / \mathrm{km}$ on average by $2020[2,3]$. This challenge pushes the boundaries of engineering and suddenly even the smallest of $\mathrm{CO}_{2}$ savings start to become valuable. The new driving cycle introduced is known as the Worldwide harmonized Light vehicles Test Cycle (WLTC), which resembles more realistic driving conditions than its predecessor, the New European Driving Cycle (NEDC). One of the key factors it includes is an extra-high speed stint and an overall average velocity of $46 \mathrm{~km} / \mathrm{h}$, in comparison to the previous $33 \mathrm{~km} / \mathrm{h}$ in NEDC. This increases the importance of aerodynamics where a 5\% reduction in aerodynamic drag on a typical passenger car would result in a reduction of $1.5 \mathrm{~g} / \mathrm{km}$ of $\mathrm{CO}_{2}$.

Additionally, the more thorough WLTP procedure requires manufacturers to report emissions for all variants of any certain car. Meaning that if a vehicle is sold with different rims and tyres, then the manufacturer needs to be aware of the changes in emissions these various combinations could cause, which have to be reported accordingly. More importantly, manufacturers would need to take that into consideration while optimizing the vehicle's aerodynamics. The interest in wheel drag changes can be noted in numerous publications describing the aerodynamic development of vehicles [4-6]. This follows many studies revolving around the aerodynamics of isolated wheels and their effects [7-10].

The vehicle exterior receives a lot of attention from aerodynamicists and designers. It begins with a concept that quickly evolves into clay models that can be tested and evaluated in the wind tunnel. This takes place early in the development process before any mechanical parts are manufactured, or even designed sometimes. This allows for optimization work to be performed on the vehicle exterior from very early design stages using both tests and simulations. However, the rims and tyres expected to be equipped on the car are usually not produced yet, and will not be until late stages of the vehicle development. This poses a conflict where the vehicle could experience a sudden change in drag when the physical tyre and rim geometries are introduced. The wheels are typically responsible for $25 \%$ of the overall vehicle drag even though they are relatively shielded [11]. For that reason, the possibility of modelling rims and tyres in Computational Fluid Dynamics (CFD) simulations to detect this change, as soon as a Computer Aided Design (CAD) representation is available, can alert the aerodynamicist to these changes before production. It could also open up the possibility to influence the rim and tyre designs to achieve lower drag figures.

Big challenges for CFD present themselves when modelling the wheels as they are rotating parts located in a highly turbulent flow environment with numerous separations and vortices. Several numerical and experimental investigations have looked into rim design and its effects on aerodynamic forces as well as flow patterns [12-15]. The numerical studies investigating rims usually included a de-featured tyre, known as slick, when comparing various rims. Experimental studies on the other hand tend to use fully detailed production tyres which results in different flow structures in the vicinity of the wheels. Interactions between the rims and tyres could be seen in some cases rendering it difficult to optimize each separately[11, 16, 17]. The rim modelling is typically simple and can be achieved by physically sliding the mesh, although it usually comes with an increase in computational costs as well as the requirement of having an unsteady simulation setup.

Modelling the tyre rotation is more difficult given its deformation under 
vehicle load and contact with the ground, as well as the fact that it changes slightly while rotating. Typically, a rotating wall boundary condition can be used for modelling slick tyres, which makes it possible to keep the mesh fixed. However, when more accurate results and experimental validation are needed, the tyre pattern effect needs to be represented, and the effects of the different pattern features need to be well understood. Experimental work on tyres have previously concluded that a more curved tyre shoulder, a smoother side wall, and finer lateral grooves are desired features for low drag tyres [18]. Although similar trends for tyre curvature effect and side wall detail sensitivity could be seen in CFD simulations, predicting the lateral grooves effects remains challenging, as a simple rotating wall boundary condition would result in over predicted drag values $[16,19]$. For that reason, and due to the fine meshing required to resolve these lateral grooves, investigations into replacing the pattern with surface roughness have been considered [20]. Also, investigations of having a fully moving mesh, by applying an immersed boundary method approach, has been implemented but with the current limitation of keeping the tyre geometry undeformed [21]. A Moving Reference Frame - grooves (MRFg) approach for modelling the lateral grooves has also been proposed in [19] and has shown promising results.

The work presented in this paper addresses many of the discussed challenges through the use of special physical tyres which are cut to desired specifications. It was thus possible to isolate the individual effects of rain grooves and lateral grooves in the wind tunnel tests. Their local and global effects are further discussed using CFD. The simulations setup sensitivity has been investigated and a sliding mesh approach is implemented for rim simulations combined with an improved MRFg approach for modelling the tyre presented in [22]. The tyre feature predictions obtained from CFD, for two sets of rims, are compared to the wind tunnel test measurements.

\section{Methodology}

In this section, a detailed description of the physical test objects and their representation in the virtual models is presented, followed by the experimental and numerical setups.

\section{Physical and Virtual Geometry}

The tests are performed on a production Volvo S60 with a modified rigid suspension, which allows for the control of the wheel centre in relation to the trim heights. By doing so, it is ensured that the wheel centre height for any testing velocity will be the same as the stationary position. This allows for matching the wheel centre position in the CFD simulations without doing any additional measurements during the test. The rigid suspension brackets added on the test object are also added in the simulations for the sake of completeness. The cooling inlets have been closed with the help of a stiff aluminium plate which was reproduced in the virtual model as well. For the same reason, the split lines along the hood, trunk and rear bumper have all been taped.

Reproducing tyre geometries accurately in simulations is one of the most challenging tasks facing aerodynamicists today, due to the complexity of the pattern and the wheel deformation due to rotation. In this work, Avon racing tyres are selected providing certain advantages which simplifies the tyre modelling challenge. These advantages mainly arise from the tyres, availability in slick condition and its high stiffness.

First, in order to ensure similar tyre profile and deformations, all tyres used in this study are initially slick tyres of the same size (210/635R17), with a lateral width $218 \mathrm{~mm}$ and a diameter of $640 \mathrm{~mm}$. The tyre dimension measurements are performed after 3D scanning of the tyres while they are mounted on the 17 " $\mathrm{x} 7$ " production rims, used in this study. The nominal inflation pressure for these tyres is 1.4 bar which is significantly lower than typical passenger car tyres. The racing oriented tyre construction delivers a very stiff tyre which preserves its profile even when inflated up to 2.6 bar, as has been observed after comparing $3 \mathrm{D}$ scans from the 1.4 and 2.6 bar pressures. Given the unaltered tyre geometry during the inflation pressure tests, even after such a large change in internal pressure, it can be assumed that the tyre geometry changes due to internal centrifugal forces from rotation can be neglected. This effect is a property of the chosen tyres and would not be the same for conventional passenger car tyres, which would experience significant deformation. However, the tyres are not completely rigid and they do deform under the weight of the car, an external force, thus resulting in bulging of the side wall close to the ground. A tyre contact patch is also formed and as expected the front contact patch is significantly larger than the rear contact patch, given the forward offset centre of gravity of the car. The contact patch has been measured in the stationary position and replicated in the simulations. The contact patch imprint is obtained using spray paint where the patch width can be identified down to a few millimetres. The patch length however is over estimated as the spray paint cannot reach into the tight gap between the tyre and ground. Yet, this is acceptable for simulation purposes as meshing this small gap is difficult and it needs to be simplified.

To create the different tyre patterns, manual cutting is performed with high accuracy and repeatability in order to obtain four total tyre patterns: slick (S), rain grooved(R), lateral grooved (G), and full pattern (D). The rain and lateral grooves geometries are simplified representations of common passenger car tyre patterns, designed within the limitations of the cutting tools and the tyre tread depth. This simplified representation allowed for manual CAD implementation of the grooves to obtain fully deformed detailed tyres, which are simple to mesh and simulate. All grooves have been implemented in CAD on the deformed slick tyre representation so that the tyre deformation is identical. The tyres are all new with a generally smooth surface finish, unlike conventional passenger car tyres. The CFD representation of the tyres and the physical tyres can be seen in Figure 1.
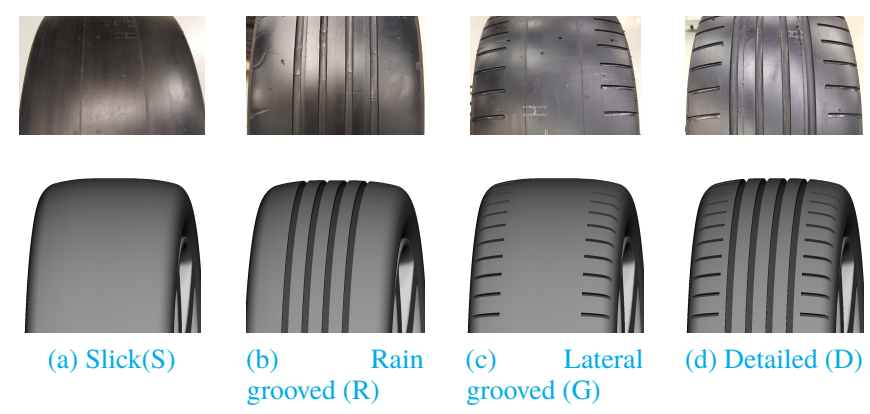

Figure 1: Investigated tyre sets: physical (top) and virtual (bottom).

All tyres are tested on an open production rim with an additional closed rim configuration, where an aluminium sheet metal is bolted on to the rim. A similar replication of the geometry is performed in CFD thus preserving the rim spokes and the internal rim geometry. A representation of the rims can be seen in Figure 2.

\section{Experimental Setup}

The tests were conducted in the full scale Volvo Cars Aerodynamic Wind Tunnel (PVT) at a speed of $100 \mathrm{kph}$. The tunnel is a closed loop type with a slotted wall test section and a cross sectional area of $27 \mathrm{~m}^{2}$. It is equipped with a five-belt moving ground system with tangential blowers 


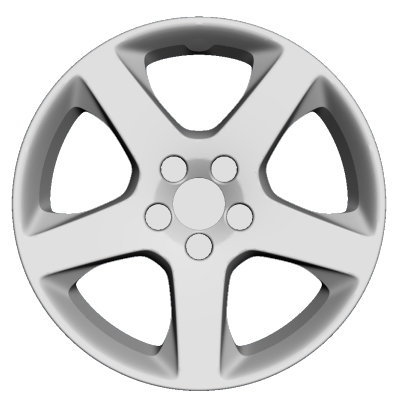

(a) Open rim (OR)

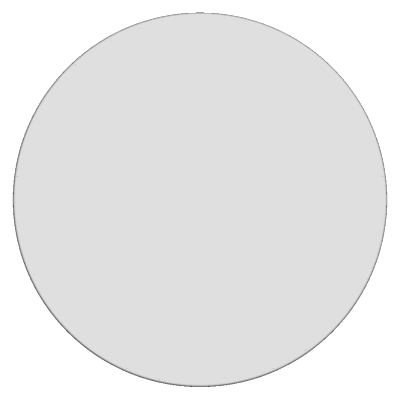

(b) Closed rim (CR)
Figure 2: Geometry representations of the rims.

to simulate road conditions. The tunnel is accredited according the European Accreditation procedure EA 4/02 and has a one count $(0.001$ $\mathrm{C}_{\mathrm{d}}$ ) measurement uncertainty for drag coefficient measurements within the same test, more details about the wind tunnel can be found in [23]. Only force measurements were performed during the test campaign. These are converted to aerodynamic coefficients, which are corrected for wake blockage, solid blockage, and horizontal buoyancy. Figure 3 shows the car in the wind tunnel.

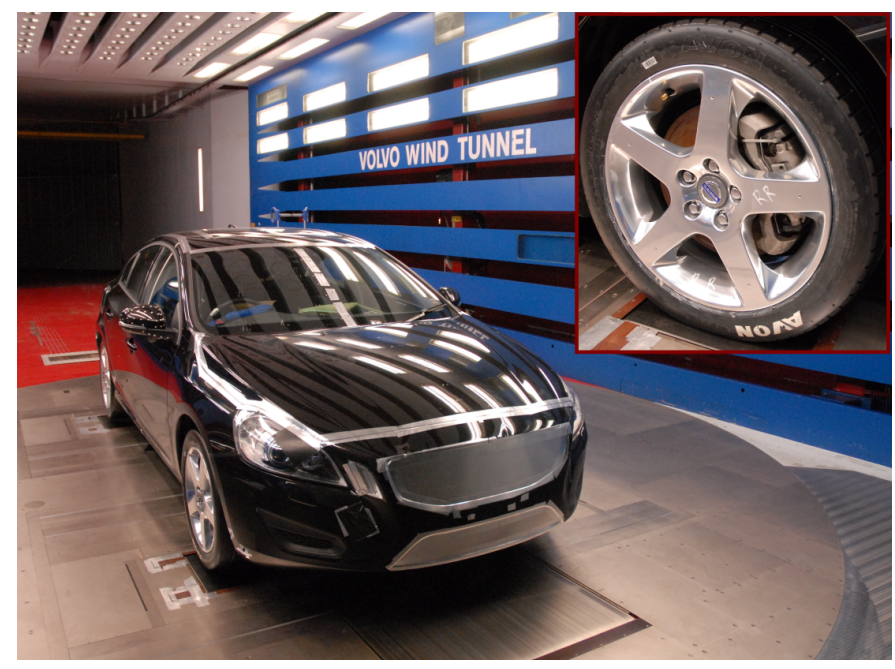

Figure 3: The test vehicle in the wind tunnel with a closer look at the wheel

\section{Numerical setup}

The simulations are performed in StarCCM+ (v11.06) using a hybrid RANS-LES turbulence modelling approach. The formulation used is the Improved Delayed Detached Eddy Simulation (IDDES)[24], with second order temporal discretization and second order upwind spatial discretization. A hybrid Gauss-Least Square Method (Gauss-LSQ) is used for gradient computation with second order accuracy. The simulations are solved up to a steady state k-w SST solution for initialization. Then, three seconds of physical time are computed with gradual reduction of the timestep in order to allow the flow field to develop smoothly. Finally, two seconds of physical time with a timestep of $2 \times 10^{-4}$ seconds are computed and averaged to obtain the mean flow solution. Thus, the wheel rotates by 1 degree per time step and performs around 28 revolutions during the averaging time.

When running an unsteady simulation it is important to choose the averaging time and time step carefully to obtain accurate results. The time step choice not only affects the sampling time but also affects the flow physics as it directly affects the Courant number (CFL). Ideally, the CFL should be below 1 in the vast majority of the domain, which would be the equivalent of a time step of $5 \times 10^{-5}$ seconds with the current mesh resolution. However, the computational costs for such a small time step are not affordable within the available computational resources for the project. Given that the flow is iterated several times within each time step, and that a second order temporal discretization is used, larger time steps could also be feasible. This is investigated by performing simulations on the reference case, closed rims and slick tyres, with multiple time step sizes and comparing the drag and lift distributions. The times step sizes investigated are: $5 \times 10^{-5}, 1 \times 10^{-4}$, $2 \times 10^{-4}$, and $4 \times 10^{-4}$ seconds. An investigation into the averaging interval is also performed by extending the two seconds interval to 4 seconds for the reference case.

All simulations are done at $100 \mathrm{kph}$ with the wheel rotation modelled using a sliding mesh approach for modelling the rim spokes rotation and an MRFg (Moving Reference Frame - grooves) approach for modelling the tyre rotation. The MRFg approach combines the rotating wall boundary condition on the tyre surface and the MRF approach inside the lateral grooves. The details of its implementation and sensitivity analysis can be found in [22]. The simulations are performed in open road conditions with a fully moving ground. The rectangular domain is $70 \mathrm{~m}$ long, $20 \mathrm{~m}$ wide, and $10 \mathrm{~m}$ high, with a velocity inlet, upstream of the car, and a pressure outlet, downstream. The car geometry includes a detailed engine bay however the cooling inlets have been sealed off for all cases. By doing so any uncertainties from modelling the cooling package and engine bay geometry are avoided.

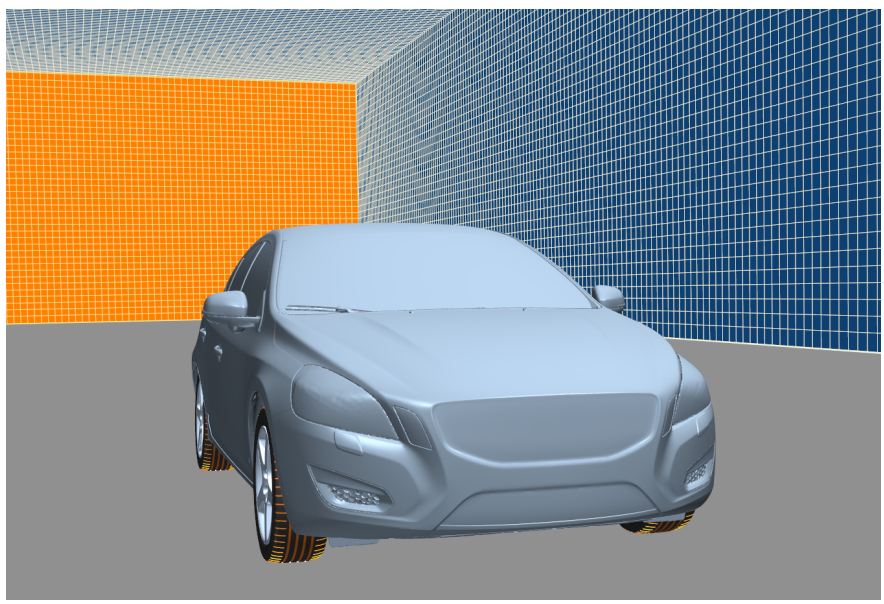

Figure 4: The virtual model in the simulation domain.

One mesh, for the complete domain, is built per rim which allows for all various tyre configurations. This ensures an identical mesh for all tyre feature investigations per rim. The mesh surface sizes varied from 1 to $8 \mathrm{~mm}$ on the vehicle, with slow growth into the domain for key sensitive areas around the car. Six to eight prism layers, with a first cell height of $0.01 \mathrm{~mm}$, are built on all exterior surfaces which are directly exposed to the main flow and are expected to have a boundary layer build up. This has also been performed on the wheels resulting in a $y+$ below one. For all other surfaces, only one prism layer with a height of $1 \mathrm{~mm}$ is built in order to reduce the mesh count while still ensuring a constant first cell height. The total mesh count ranged around 120 million cells, requiring around 30 hours per simulation running on 2000 cores.

\section{Results and Discussion}

The CFD methodology investigation results are presented in this section. Then the CFD prediction capability for tyre modifications is compared 
to the wind tunnel predictions.

\section{CFD Methodology Investigation}

The effects of changing time step size on overall drag and lift coefficients can be seen in Figure 5, where the forces are averaged for 2 seconds. They are presented in terms of deltas to the reference setup with $5 \times 10^{-5}$ seconds. The overall drag and lift predictions do not change significantly for time steps of $1 \times 10^{-4}$ and $2 \times 10^{-4}$ seconds, while $4 \times 10^{-4}$ deviate from the overall drag and front lift values. Thus, for the most time efficient approach, the $2 \times 10^{-4}$ seconds time step is preferable as it reduces the simulation time by $60 \%$ from the $5 \times 10^{-5}$ s time step, as shown in Figure 6 . The increase in time step is not linearly related to the time saving since it is accompanied with an increase in inner iterations in order to get a converged flow field within the time step.

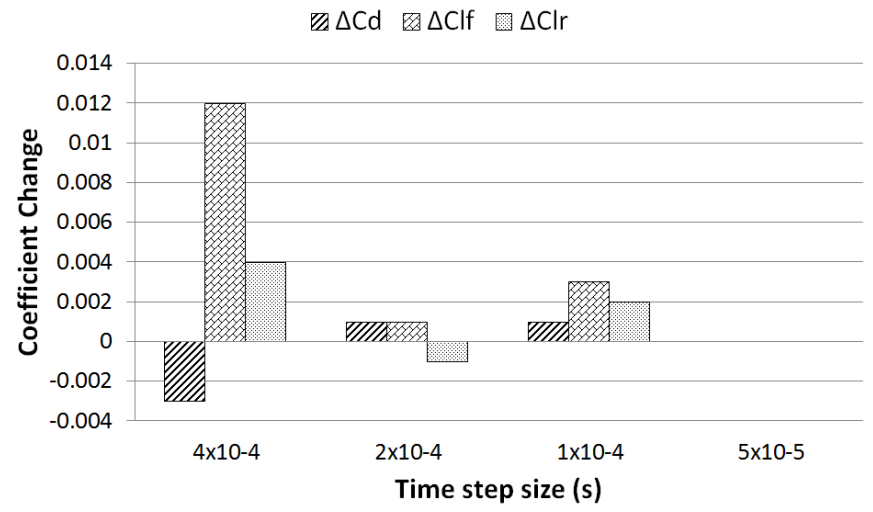

Figure 5: Changes in drag and lift distribution when varying the time step relative to $5 \times 10^{-5} \mathrm{~s}$.

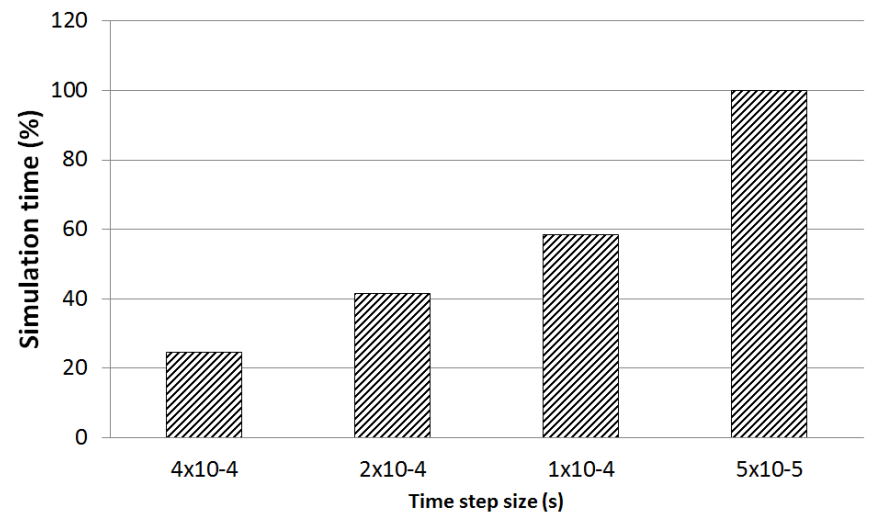

Figure 6: Changes in simulation solving time presented in percentage relative to $5 \times 10^{-5} \mathrm{~s}$

However, prediction of the overall drag value is not a sufficient measure as the distribution over the vehicle must also be similarly predicted in order to enable reliable local optimization. This is investigated by plotting the cumulative drag difference over the vehicle in order to confirm that the drag development over the length of the vehicle is close. The cumulative drag is obtained by summing the local drag contribution for each vehicle surface cell along the length of the car (x-direction).

Figure 7 presents the deltas of cumulative drag and lift development along the length of the vehicle for the case of $2 \times 10^{-4}$ vs $5 \times 10^{-5}$ seconds time steps. It can be seen that at no point along the vehicle length does the drag or lift deviate by significant values. This further confirms that a time step of $2 \times 10^{-4}$ is sufficient for the simulation setup.

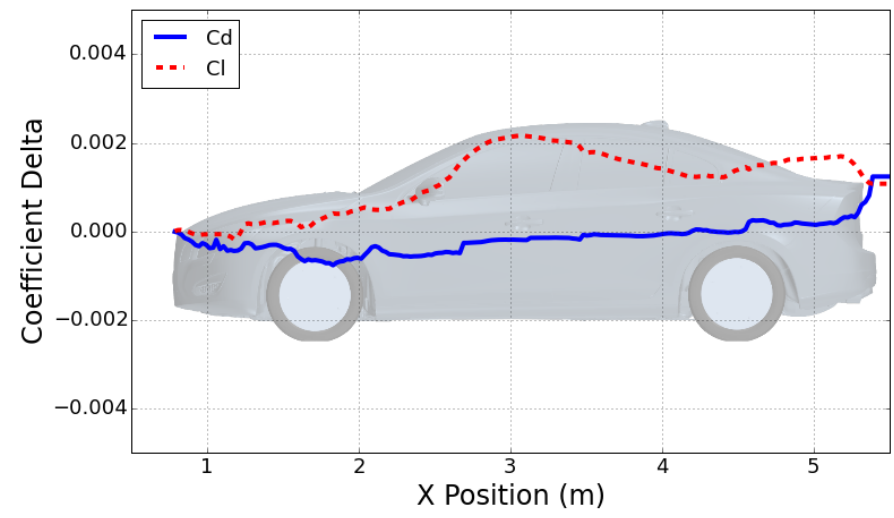

Figure 7: The difference in aerodynamic coefficients along the length of the car for a $2 \times 10^{-4}$ vs $5 \times 10^{-5} \mathrm{~s}$

With the time step set, the effect of averaging time on the results is investigated by extending the averaging interval from two seconds to four seconds. The overall drag and lift values, as well as the cumulative drag and lift, showed no significant deviations and this can be seen from the cumulative $C_{d}$ and $C_{l}$, along the vehicle length, plots shown in Figure 8 .

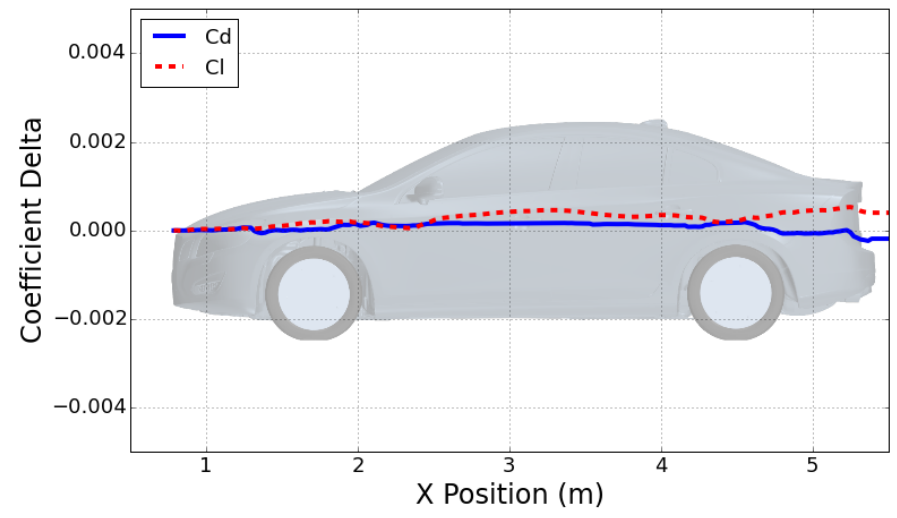

Figure 8: The difference in aerodynamic coefficients along the length of the car for a $2 \mathrm{~s}$ vs $4 \mathrm{~s}$ averaging time.

An analysis of looking into the flow quantities has also been performed by 3D mapping the complete time averaged flow fields and analysing the deviations. It has been noted that small deviations in velocity of the order of $1 \mathrm{~m} / \mathrm{s}$ can be seen in the rear wake area of the car. This is not surprising given that the rear wake frequency usually is of the order of several Hertz, and hence a time averaging of $2 \mathrm{~s}$ might capture a relatively small number of cycles. However, these deviations showed very little effect on the pressure, less that $5 \mathrm{~Pa}$ deviations in the flow field with almost no deviations on the vehicle surface. Given this general match in flow field comparison, the 2 seconds averaging time interval predicts the numerical results with acceptable accuracy, with a little over half the computational resources.

\section{Tyre Feature: rain grooves}

The changes in drag resulting from adding rain grooves to slick and lateral grooved tyres on open and closed rims can be seen in Figure 9. Whenever a significant trend is present, for the closed rim cases (CR-S 
and CR-G), as well as the open rim with lateral grooved tyre (OR-G), it is predicted in the same direction with a similar magnitude. For the open rim with slick tyre, OR-S, the drag changes are too small for any strong conclusions to be drawn. The drag reduction in CFD can be traced to the

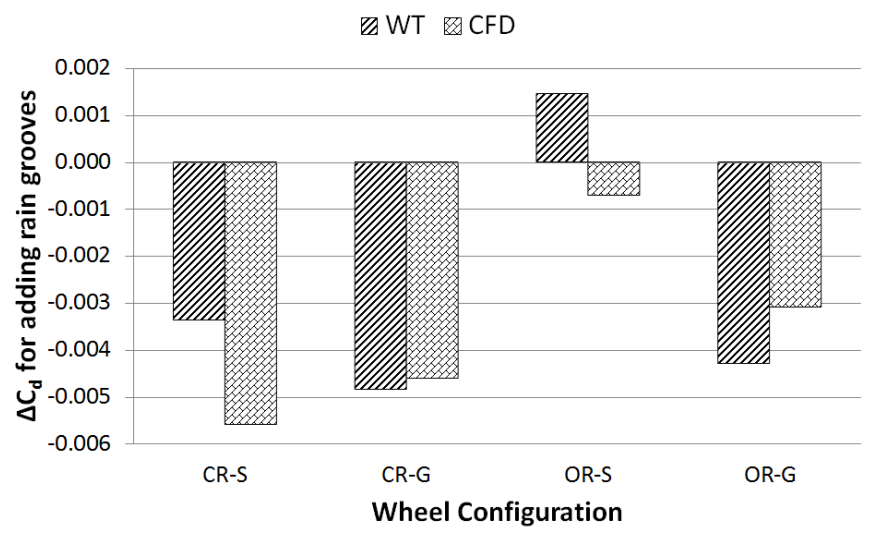

Figure 9: The change in drag when introducing rain grooves on various wheel configurations from wind tunnel tests and CFD simulations.

rear wheels and the base area of the car. This was unexpected since it has been previously observed that the rain grooves reduce the stagnation pressure on the front tyre and equalize the low pressure behind it as it connects the two areas, [16]. However, the flow around the underbody seems to change and any benefits added from this effect are equalized by increases in drag elsewhere around the front wheel. This can be seen in Figure 10, where, despite the fluctuations around the front wheel, the drag difference between the rain grooved tyre and the slick tyre is null at the middle of the car. Further downstream the drag reduction is seen around the rear wheel area leading to a sharp reduction around the trunk and base.

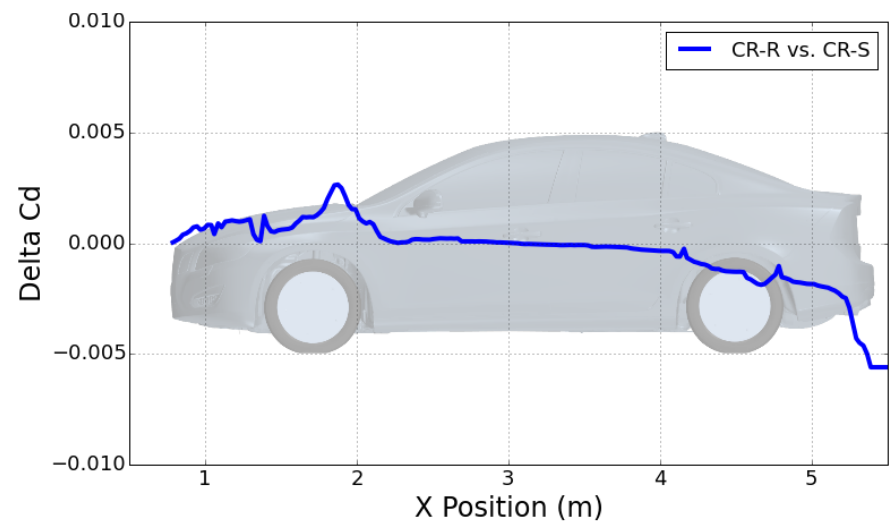

Figure 10: The change in drag along the length of the car when introducing rain grooves on a closed rim wheel with slick tyres.

Figure 11 shows the mean base pressure for the slick and rain grooved tyres on a closed rim. The base pressure is higher when the rain grooves are present as shown in Figure 11b compared to Figure 11a.

Figure $11 \mathrm{~b}$ also shows two strong low pressure spots on the lower outer edge of the rear wheels, indicating a stronger inflow. It is the result of a vortex created behind the rear wheel which moves towards the rear wake and can be seen in Figure 12.

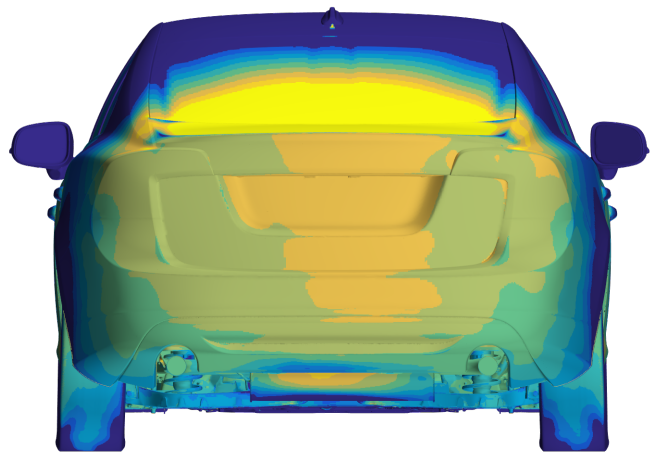

(a) Closed rim slick (CR-S)

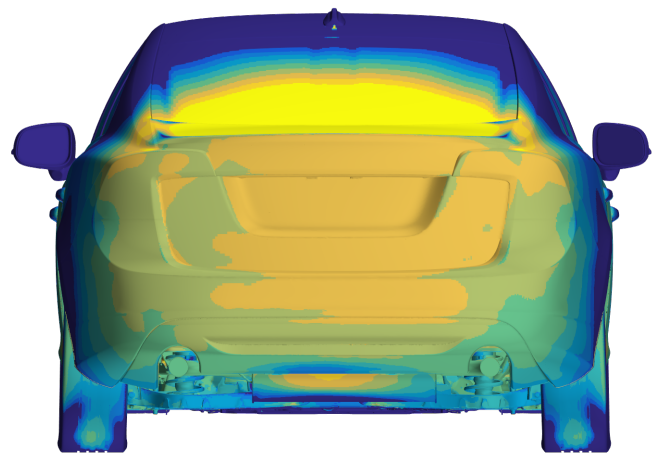

(b) Closed rim rain grooved (CR-R)

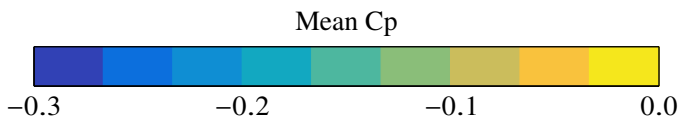

Figure 11: Mean pressure coefficient distribution on the base of the car for different wheel configurations.

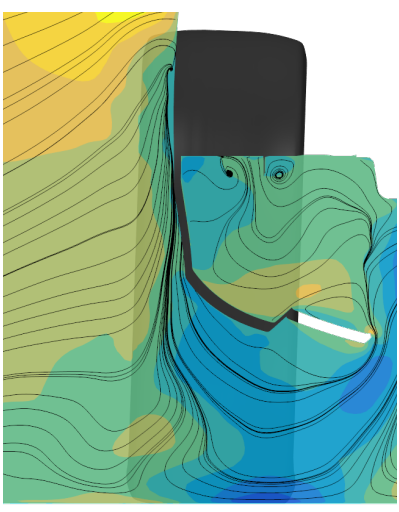

(a) Closed rim slick (CR-S)

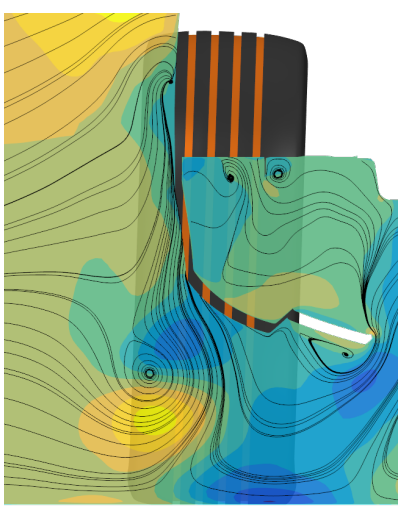

(b) Closed rim rain grooved (CR-R)

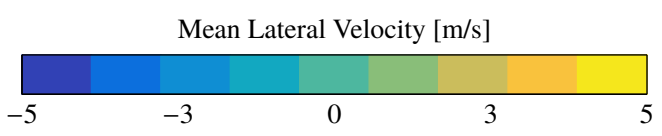

Figure 12: Mean lateral velocity in a plane $0.5 \mathrm{~m}$ behind the rear wheel centre with in-plane streamlines.

\section{Tyre feature: lateral grooves}

The changes in drag resulting from adding lateral grooves to slick and rain grooved tyres on open and closed rims can be seen in Figure 13. For the cases with the closed rim, CR-S and CR-R, the CFD prediction is not correct. 
$叉 W T \otimes C F D$

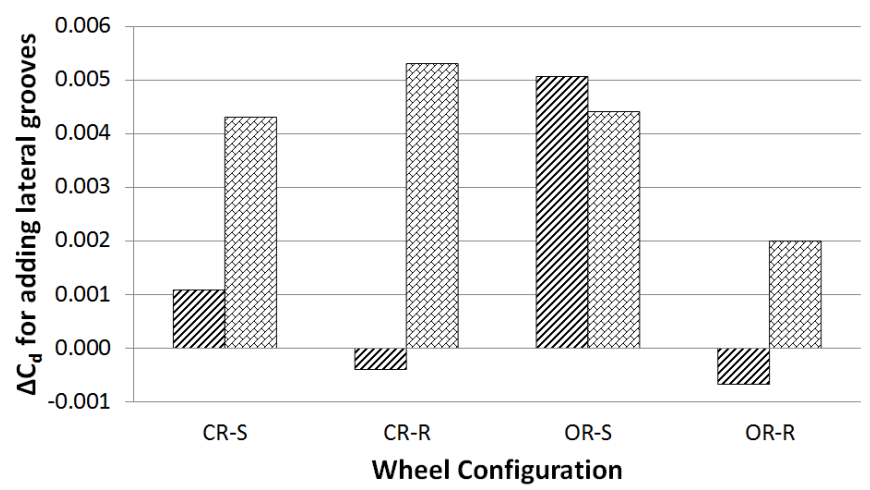

Figure 13: The change in drag when introducing lateral grooves on various wheel configurations from wind tunnel tests and CFD simulations.

The explanation for the discrepancy for both closed rim cases is not a hundred percent clear. CFD seems to either over predict the drag with the lateral grooves present, or under predict the drag when they are not. The latter is the most likely scenario since, in reality the tyre shoulder is a rough surface and not as smooth as simulated, and surface roughness in such a sensitive area with high velocities and steep gradients could alter the flow significantly if a separation is triggered. With the rim being completely closed, the tyre shoulder will be the main factor determining the flow behaviour around the wheel. The discrepancy in results between CFD and experiments is significantly reduced with the open rim, OR-S and OR-R, given the interaction with the rim spokes which alters and likely dominates the flow in the close vicinity of the wheel. The effect the rim introduces can be seen when looking at q-criterion around the front left wheel, presented in Figure 14. The closed rim introduces very few vortex structures, Figure 14a, while the open rim generates a small sheet of vortices which covers most of the lower rim part, Figure 14b.

It is also worth noting that the tyre side wall in the simulations is completely smooth unlike the test object which contains some protruding text spread in a sparse manner. This could also contribute to a slight under prediction of the drag for the closed rim configurations with slicks. The effect of this side writing would become less pronounced once the lateral grooves are added as they introduce turbulent structures and vortices into the flow upstream of the side wall.

The drag increase from adding the lateral grooves in CFD has been directly linked to the front wheels without any major effects resulting from the rear wheels. This is seen when plotting the change in cumulative drag over the length of the vehicle. This is shown in Figure 15 for OR-S. When adding the lateral grooves a sharp increase in drag takes place around the front wheels, however when the flow stabilizes downstream of the front wheels this increase in drag is not affected any more. Similar behaviour has been observed with all configurations pointing to very little change around the rear wheels.

The small vortices generated from the lateral grooves merge in the contact patch separation, Figure 14c, and its imprint can be seen by looking at the mean total pressure coefficient shown in Figure 16. The outer low pressure region at the front tyre contact patch, is taller when the lateral grooves are added. This can be seen when comparing Figure 16c to Figure 16a which are located in a plane going through the front wheel centre at $x=1.7$. Further downstream half a meter behind the front wheel centre, at $x=2.2$, this low pressure region stretches out in width while moving away from the vehicle, which explains the lack of further interactions downstream.

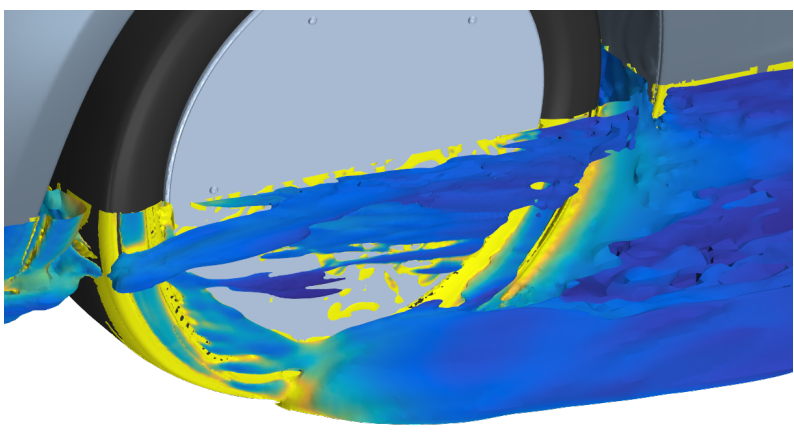

(a) Closed rim slick (CR-S)

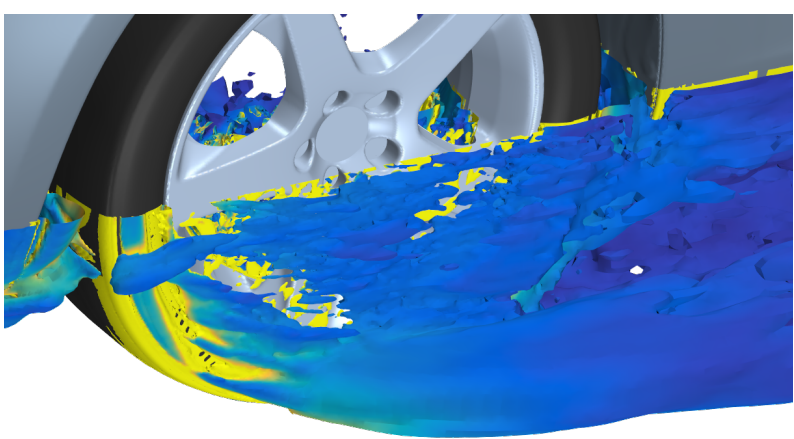

(b) Open rim slick (OR-S)

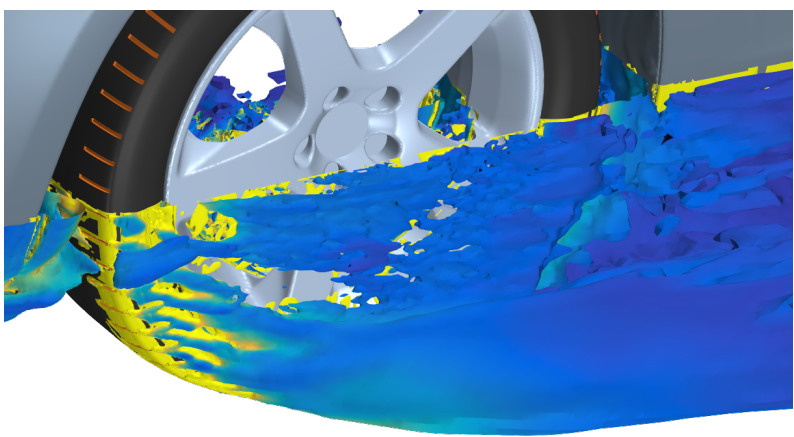

(c) Open rim lateral grooved (OR-G)

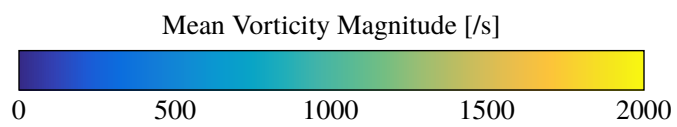

Figure 14: Isosurface of Q-criterion at $5000 / \mathrm{s}^{2}$ coloured by mean vorticity magnitude around the front left wheel.

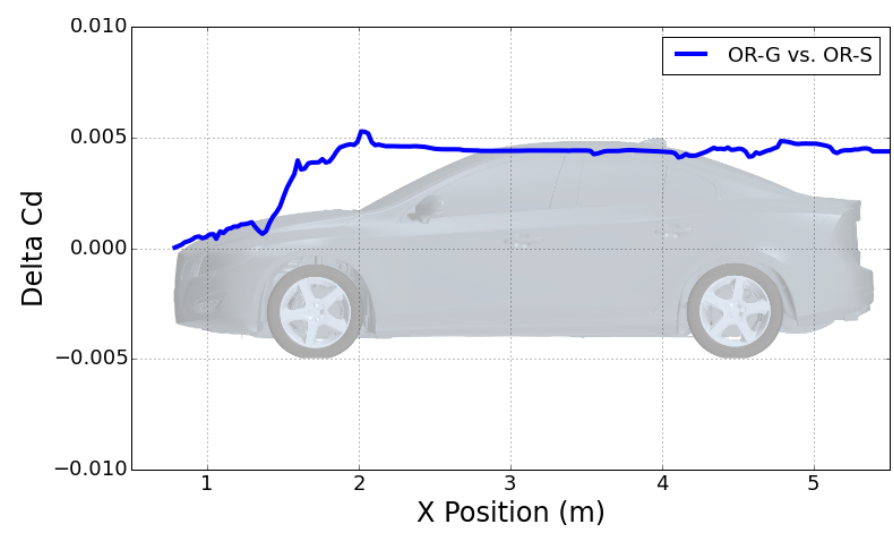

Figure 15: The change in drag along the length of the car when introducing lateral grooves on an open rim wheel with slick tyres. 


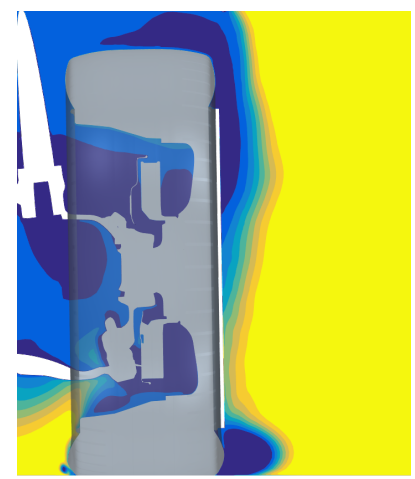

(a) OR-S at $\mathrm{x}=1.7$

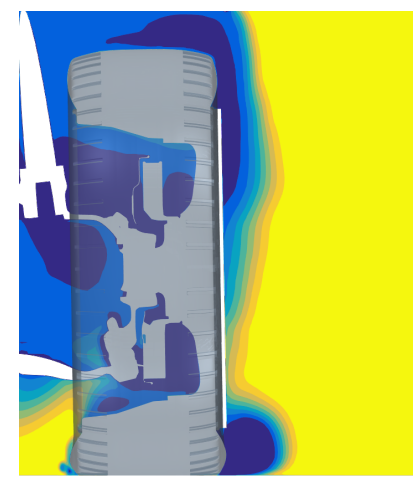

(c) OR-G at $x=1.7$

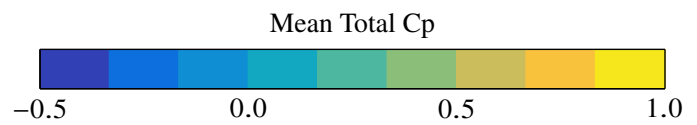

Figure 16: Mean total pressure coefficient around the front left wheel in a plane at the front wheel center $(\mathrm{x}=1.7)$ and $0.5 \mathrm{~m}$ downstream $(\mathrm{x}=2.2)$.

\section{Tyre feature: detailed pattern}

The effects of adding the complete detailed pattern are summarized in Table 1 . The wind tunnel tests show that the drag is reduced by $0.004 \mathrm{C}_{\mathrm{d}}$ when a pattern is added on the tyre for the closed rim case. A negligible increase of $0.001 \mathrm{C}_{\mathrm{d}}$ can be noted for the open rim case. This result has previously been noted by Wickern et al. [11] with $0.005 \mathrm{C}_{\mathrm{d}}$ reduction and $0.002 \mathrm{C}_{\mathrm{d}}$ increase even though the test object, wheels, and tyres are completely different. It is also worth highlighting that the experimental results presented by Wickern et al. [11] were obtained in a very different experimental setup. The wind tunnel was equipped with a fully moving ground and with the vehicle suspended from a sting support through the rear with an internal balance. Therefore it is likely that the reduction in drag for the closed rim case is independent of the wind tunnel's moving ground systems.

Table 1: The change in drag when adding the detailed pattern on a slick tyre for closed and open rim configurations.

\begin{tabular}{rrrr}
\hline & WT & CFD & Wickern et al. [11] \\
\hline CR & -0.004 & 0.000 & -0.005 \\
OR & 0.001 & 0.001 & 0.002 \\
\hline
\end{tabular}

The wind tunnel results are predicted well in CFD with the open rim case, however the closed rim case is not. Again, when simulating with a closed rim, it is not clear if the detailed tyre configuration is being over predicted or if the slick tyre case has been under predicted but the latter is most likely the case.

\section{Comparison to slick}

The effects of adding the different grooves separately, as well as the combined effect, on a slick tyre on both open and closed rims are presented. To sum up the results, the predicted change in drag from the simulations for any specific configuration is subtracted by the respective measured change in the wind tunnel test. It can be written as

$$
\Delta \Delta C_{d \text { conf }}=\left(C_{d \text { conf }}-C_{d \text { Slick }}\right)_{C F D}-\left(C_{d \text { conf }}-C_{d \text { Slick }}\right)_{W T}
$$

where $C_{d \text { conf }}$, is the drag for the configuration of interest and $C_{d}$ Slick is the drag when changing to a slick. This results in a direct comparison of the prediction capability of CFD compared to the wind tunnel measurements. The capabilities are summarized in Figure 17 with a dashed line highlighting the uncertainty margin. This margin is based on the $0.001 \mathrm{C}_{\mathrm{d}}$ experimental repeatability uncertainty and a $0.002 \mathrm{C}_{\mathrm{d}}$ simulation uncertainty estimate from the CFD methodology investigation.

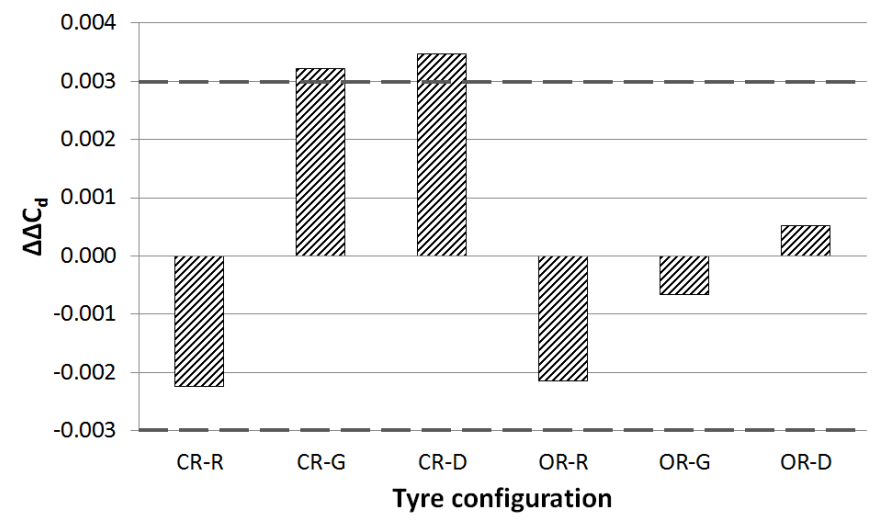

Figure 17: $\Delta \Delta C_{d}$ for various wheel configurations demonstrating the difference between the CFD prediction of tyre feature effects compared to the measured wind tunnel values.

For the open rim cases, OR-R, OR-S, and OR-D, as well as the closed rim case, CR-R, the prediction is well within the uncertainty margins. However, for the closed rim cases, CR-G and CR-D, the results lie slightly outside the uncertainty margin. This is not surprising as it has previously been discussed, in the lateral grooves section, that the closed rim case has shown an unexpected increase in drag when the lateral grooves are added.

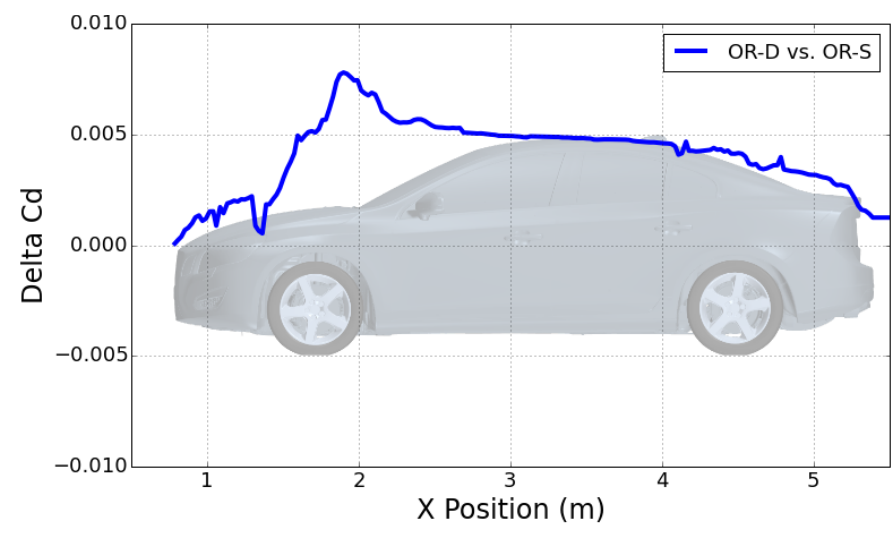

Figure 18: The change in drag along the length of the car when introducing detailed pattern on an open rim wheel with slick tyres. 
Figure 18 shows the cumulative $\mathrm{C}_{\mathrm{d}}$, along the length of the car, difference between detailed and slick pattern on an open rim. The increase is drag around the front wheel is a result from both the lateral and rain grooves. As described previously in the lateral grooves section, the same effect of enlarged contact patch separation occurs and can be seen when comparing Figure 19a to Figure 16a. The low pressure region also stretches in width as the flow moves downstream. As the rain grooves are present in this case, the flow is slightly altered leading to an increase in pressure behind the tyre at the ground yet a reduction in pressure around the side skirts, seen in Figure 19b. These effects seem to cancel out resulting in a 0.005 net increase in $C_{d}$ at $x=3 \mathrm{~m}$, similar to the case of the open rim with lateral grooved tyre (OR-G), Figure 15.

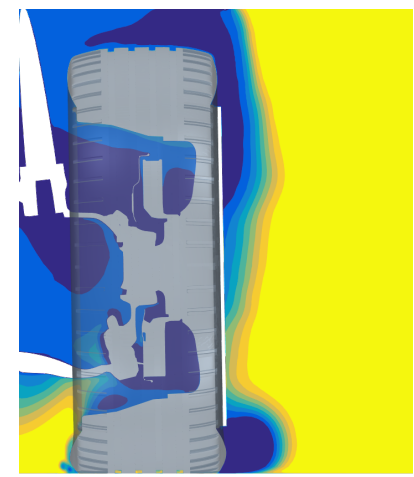

(a) OR-D at $x=1.7$

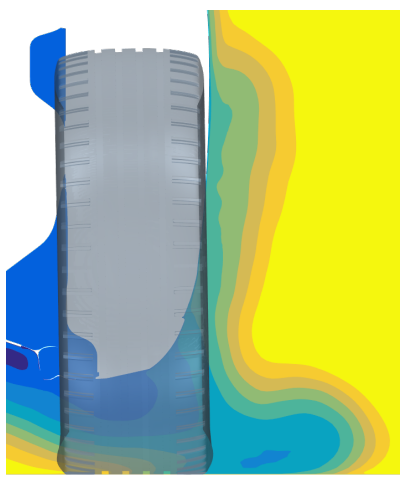

(b) OR-D at $x=2.2$

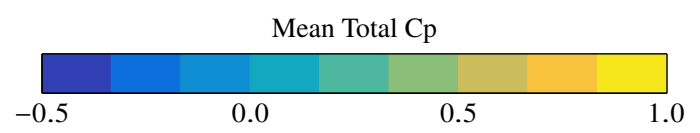

Figure 19: Mean total pressure coefficient around the front left wheel in a plane at the front wheel centre $(x=1.7)$ and $0.5 \mathrm{~m}$ downstream $(\mathrm{x}=2.2)$

As the flow travels further downstream a reduction in drag occurs at the rear wheels and the base resulting in only $0.001 \mathrm{C}_{\mathrm{d}}$ increase overall. The slightly higher base pressure can be seen in Figure 20 accompanied with an increased pressure on the muffler.

\section{Ventilation moment}

Another interesting factor that changes with the addition of tyre details is the ventilation moment, the aerodynamic resistance to the wheel's rotation. Although difficult to measure in the wind tunnel where it needs to be isolated from other losses, like rolling resistance, this is easily calculated in CFD. The ventilation moment has been output for each time step and the average for the front left and rear left wheels can be seen in Figure 21. The ventilation moments on the right wheels were similar to the left wheels, hence the detailed results are only presented for one side of the car.

As expected, the ventilation moment on the open rim configurations is significantly higher than the closed rim, which matches well with earlier studies on the subject $[25,26]$. It could also be seen that the ventilation moments for the front wheel, Figure $21 \mathrm{a}$, are consistently higher than the rear wheel, Figure 21b. This can be explained by the fact that the front wheel is more exposed to the on coming flow. When the rim is closed and the tyres are slicks, the ventilation drag is at its lowest at $0.53 \mathrm{~N} \cdot \mathrm{m}$, front, and $0.50 \mathrm{~N} \cdot \mathrm{m}$, rear. However when the rim is opened, the spokes interact more with the flow and the ventilation moments increase by $0.22 \mathrm{~N} \cdot \mathrm{m}$ in the front, and $0.2 \mathrm{~N} \cdot \mathrm{m}$ in the rear.

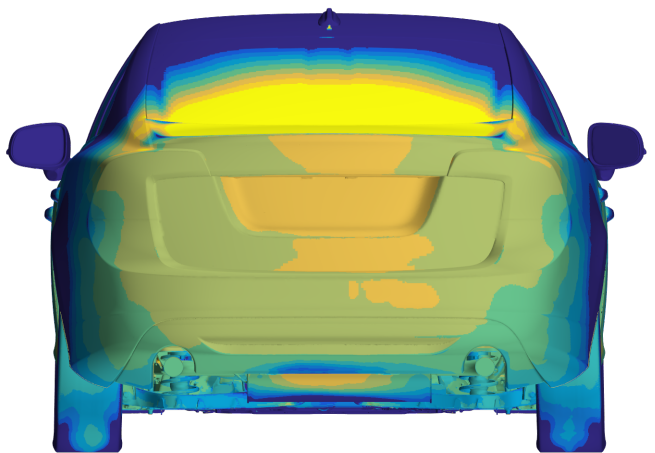

(a) Open rim slick (OR-S)

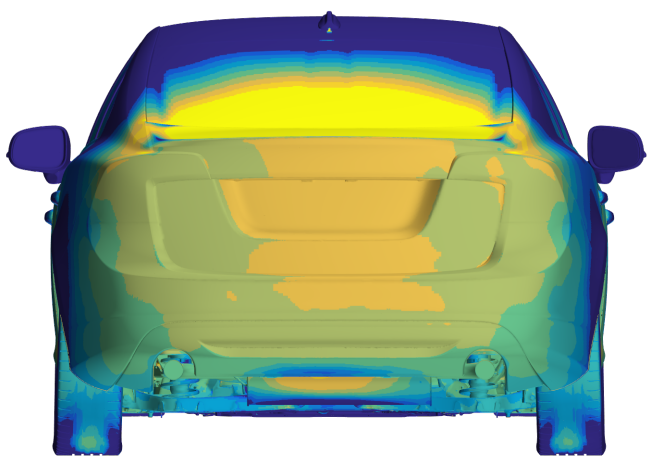

(b) Open rim detailed (OR-D)

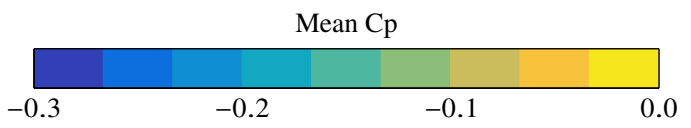

Figure 20: Mean pressure coefficient distribution on the base of the car for different wheel configurations.

Adding the tyre features also showed significant changes in ventilation moments. This is mainly attributed to the lateral grooves and can be seen when comparing the lateral grooved tyres $(\mathrm{G})$ to rain grooved tyres $(\mathrm{R})$ and slicks (S). Also due to the front wheel being more exposed, the moment increase from tyre features is more pronounced on the front wheel than the rear wheel. The rain grooves showed almost no change in ventilation moment on the rear wheel for both rim configurations. The changes in ventilation moments for the different tyre configurations were very consistent independent of the rim design.

\section{Conclusion}

This work demonstrates the ability of CFD simulations using MRFg approach to predict tyre feature effects. A study of the effects of time step size and averaging time is presented to motivate the numerical setup. Overall, the drag change for tyre features was well predicted in the open rim case. However, the closed rim case proved to be more challenging with the effects of lateral grooves slightly outside of uncertainty margins.

The following conclusions can be made:

- An averaging interval of 2 seconds with a time step of $2 \times 10^{-4}$ seconds has proven to be sufficient to produce accurate results in terms of drag and lift prediction.

- The rain grooves effect is generally well predicted in simulations after comparison to wind tunnel results. The reduction in drag they introduce could be seen in CFD around the rear wheels and on base pressure. 

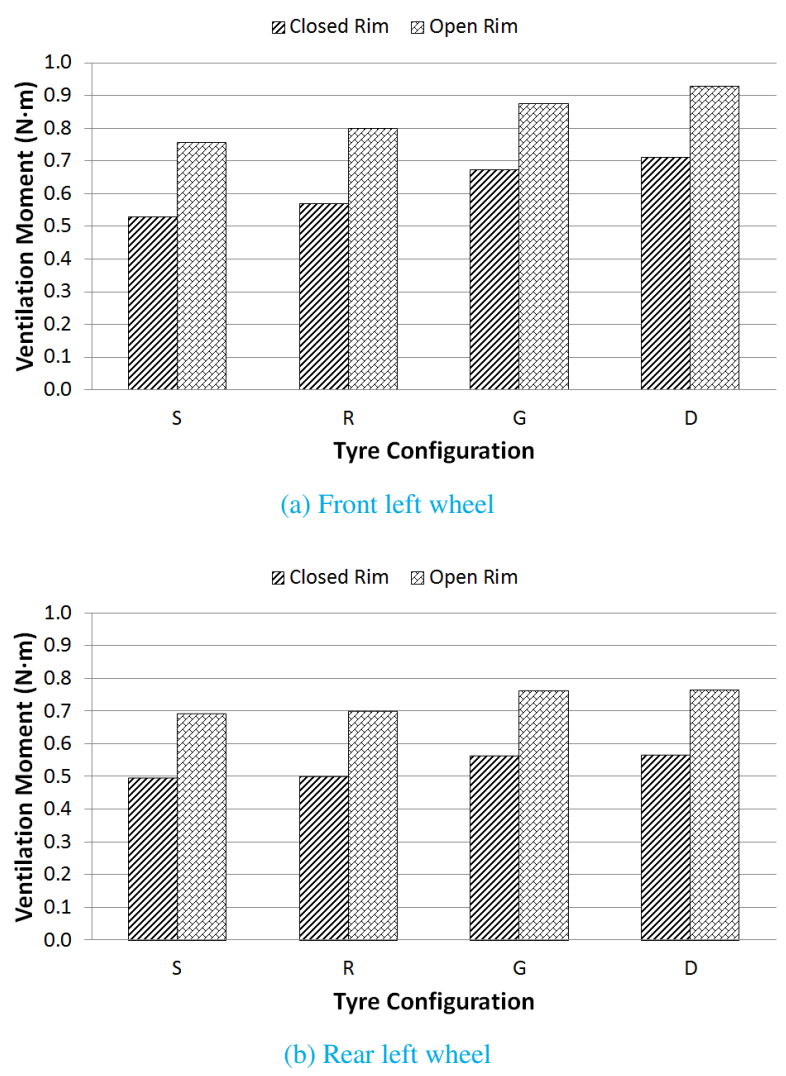

Figure 21: The ventilation moments on the front left and rear left wheels for different tyre configurations with open and closed rims.

- In CFD, the lateral grooves result in an increase in drag around the front wheels without additional interactions further downstream. This increase did not match the wind tunnel results for the closed rim configurations.

- Based on the experimental results, a detailed tyre could have lower drag than a slick depending on the rim design.

- From CFD, the ventilation moment increases when the tyre details are added, especially the lateral grooves. It has been observed that the increase on the front wheel is larger in magnitude than on the rear wheel.

\section{Future Work}

In the future, the comparison between CFD and WT tests will be complemented by further flow field analysis. This requires performing pressure and velocity measurements in planes in the vicinity of the wheels. Ideally, the wind tunnel geometry could also be included in the CFD simulations in order to reproduce the effects of the five-belt moving ground system, struts, and tunnel blockage effect on the flow field. This would allow to identify interference effects in the experiments resulting from the boundary layer control system.

The side wall details will be investigated to identify its influence, if any, on the inaccurate predictions of the closed rim design.

Investigations of these effects on different vehicle shapes will be performed, in order to check whether the observations reported in this work, regarding tyre pattern effects, are vehicle dependent.

\section{References}

[1] European Environment Agency, "National emissions reported to the UNFCCC and to the EU Greenhouse Gas Monitoring Mechanism",

http ://www. eea . europa . eu/data - and - maps/data/national emissions-reported-to-the-unfccc-and-to-the-eu-greenhousegas-monitoring-mechanism-13, 2017.

[2] Council of European Union, "Regulation (EC) 443/2009 of the European Parliament and of the Council setting emission performance standards for new passenger cars as part of the Community's integrated approach to reduce CO 2 emissions from light-duty vehicles", http://data.europa.eu/eli/reg/2009/443/2013-05-08, 2013.

[3] Council of European Union, "Regulation (EU) No 333/2014 of the European Parliament and of the Council of 11 March 2014 amending Regulation (EC) No 443/2009 to define the modalities for reaching the 2020 target to reduce CO 2 emissions from new passenger cars", http://data.europa.eu/eli/reg/2014/333/oj, 2014.

[4] D'Hooge, A., Palin, R. B., Johnson, S., Duncan, B., and Gargoloff, J. I., "The Aerodynamic Development of the Tesla Model S - Part 2: Wheel Design Optimization”, SAE 2012 World Congress \& Exhibition, (SAE International, Apr. 2012), DoI: 10.4271/2012-01-0178.

[5] Blacha, T. and Islam, M., "The Aerodynamic Development of the New Audi Q5", SAE International Journal of Passenger Cars Mechanical Systems 10(2): 638-648, Mar. 2017, ISSN: 1946-4002, DOI: $10.4271 / 2017-01-1522$.

[6] Chaligne, S., Turner, R., and Gaylard, A., "The aerodynamics development of the New Land Rover Discovery", 11th FKFS Conference-Progress in Vehicle Aerodynamics and Thermal Management, (Stuttgart, 2017).

[7] Cogotti, A., "Aerodynamic characteristics of car wheels", Int. Journal of Vehicle Design: 173-196, 1983.

[8] Axon, L., Garry, K., and Howell, J., "An Evaluation of CFD for Modelling the Flow Around Stationary and Rotating Isolated Wheels", Paper No: 980032, (Warrendale and PA, SAE International, 1998), DOI: $10.4271 / 980032$.

[9] Dimitriou, I. and Klussmann, S., "Aerodynamic Forces of Exposed and Enclosed Rotating Wheels as an Example of the Synergy in the Development of Racing and Passenger Cars", Paper No: 2006-01-0805, (Warrendale and PA, SAE International, 2006).

[10] Sprot, A. J., Sims-Williams, D. B., and Dominy, R. G., "The Aerodynamic Characteristics of a Fully Deformable Formula One Wind Tunnel Tyre", SAE International Journal of Passenger Cars - Mechanical Systems 5(2): 1026-1041, Apr. 2012, IssN: 1946-4002, DOI: 10.4271/2012-01-1166.

[11] Wickern, G., Zwicker, K., and Pfadenhauer, M., "Rotating Wheels - Their Impact on Wind Tunnel Test Techniques and on Vehicle Drag Results", SAE International Congress and Exposition, (SAE International, Feb. 1997), Dor: 10.4271/970133.

[12] Wäschle, A., "The Influence of Rotating Wheels on Vehicle Aerodynamics - Numerical and Experimental Investigations", SAE World Congress \& Exhibition, (SAE International, Apr. 2007), DOI: 10.4271/2007-01-0107.

[13] Landström, C., Löfdahl, L., Josefsson, L., and Walker, T., “An experimental investigation of wheel design parameters with respect to aerodynamic drag", 8th FKFS Conference-Progress in Vehicle Aerodynamics and Thermal Management, (Stuttgart, 2011). 
[14] Koitrand, S., Gaylard, A., and Fiet, G. O., "An Investigation of Wheel Aerodynamic Effects for a Saloon Car", Progress in Vehicle Aerodynamics and Thermal Management: Proceedings of the 10th FKFS-Conference, Reihe Technik, (2015), ISBN: 9783816933229.

[15] Haag, L., Blacha, T., and Indinger, T., "Experimental Investigation on the Aerodynamics of Isolated Rotating Wheels and Evaluation of Wheel Rotation Models Using Unsteady CFD", International Journal of Automotive Engineering 8(1): 7-14, 2017, DOI: 10.20485/jsaeijae.8.1_7.

[16] Hobeika, T., Sebben, S., and Landstrom, C., "Investigation of the Influence of Tyre Geometry on the Aerodynamics of Passenger Cars", SAE Int. J. Passeng. Cars - Mech. Syst. 6: 316-325, 2013, ISSN: 1946-4002, DOI: 10.4271/2013-01-0955.

[17] Landstrom, C., Josefsson, L., Walker, T., and Lofdahl, L., "Aerodynamic Effects of Different Tire Models on a Sedan Type Passenger Car", SAE Int. J. Passeng. Cars - Mech. Syst. 5: 136-151, Apr. 2012, DoI: 10.4271/2012-01-0169.

[18] Wittmeier, F., Kuthada, T., Widdecke, N., and Wiedemann, J., "Model Scale Based Process for the Development of Aerodynamic Tire Characteristics", SAE Technical Paper, (SAE International, Apr. 2014), DOI: 10.4271/2014-01-0585.

[19] Hobeika, T., Sebben, S., and Lofdahl, L., "Study of Different Tyre Simulation Methods and Effects on Passenger Car Aerodynamics", International Vehicle Aerodynamics Conference 2014, (Holywell Park, Loughborough, UK, 2014): 187-195, ISBN: 978-0-08-100199-8.

[20] Schnepf, B., Schütz, T., and Indinger, T., "Further Investigations on the Flow Around a Rotating, Isolated Wheel with Detailed Tread Pattern", SAE Int. J. Passeng. Cars - Mech. Syst. 8: 261-274, Apr. 2015, DoI: 10.4271/2015-01-1554.

[21] Lew, C., Gopalaswamy, N., Shock, R., Duncan, B., and Hoch, J., "Aerodynamic Simulation of a Standalone Rotating Treaded Tire", WCX17: SAE World Congress Experience, (SAE International, Apr. 2017), DOI: 10.4271/2017-01-1551.

[22] Hobeika, T. and Sebben, S., "CFD Investigation on Wheel Rotation Modelling", Journal of Wind Engineering and Industrial Aerodynamics 174C: 241-251, 2018, ISSN: 0167-6105.

[23] Sterneus, J., Walker, T., and Bender, T., "Upgrade of the Volvo Cars Aerodynamic Wind Tunnel”, SAE Technical Paper, (Detroit, Michigan, SAE International, 2007), DOI: 10.4271/2007-01-1043.

[24] Shur, M. L., Spalart, P. R., Strelets, M. K., and Travin, A. K., "A hybrid RANS-LES approach with delayed-DES and wall-modelled LES capabilities", International Journal of Heat and Fluid Flow 29(6): 1638-1649, 2008, ISSN: 0142-727X, DOI: 10.1016/j.jheatfluidflow.2008.07.001.

[25] Bonitz, S., "An investigation into the aerodynamic ventilation drag incurred by wheel rotation on a passenger car, and its influence on the total road load of the car.", Master's Thesis, Berlin, Germany: TU Berlin, 2012.

[26] Mayer, W. and Wiedemann, J., "The Influence of Rotating Wheels on Total Road Load", SAE World Congress \& Exhibition, (SAE International, Apr. 2007), Dor: 10.4271/2007-01-1047.

\section{Contact Information}

Assoc. Prof. Simone Sebben

Road Vehicle Aerodynamics Group

Division of Vehicle Engineering and Autonomous Systems

Department of Mechanics and Maritime Sciences
Chalmers University of Technology, Sweden

Tel. +46 (0)70-389 1176

simone.sebben@chalmers.se

Mr. Teddy Hobeika

Road Vehicle Aerodynamics Group

Division of Vehicle Engineering and Autonomous Systems

Department of Mechanics and Maritime Sciences

Chalmers University of Technology, Sweden

Tel. +46 (0)70-0738996

hobeika.teddy@gmail.com

\section{Acknowledgements}

The simulations were performed on resources provided by the Swedish National Infrastructure for Computing (SNIC) at PDC Center for High Performance Computing (PDC-HPC).

The authors would also like to thank Volvo Cars for providing access to their test facility and resources.

\section{Funding}

This work is funded by FFI (Fordonsstrategisk Forskning och Innovation, Strategic Vehicle Research and Innovation) through Energimyndigheten (Swedish Energy Agency) project number 37195-1.

\section{Nomenclature}

\begin{tabular}{ll}
\hline Symbol & Definition \\
\hline CFD & Computational Fluid Dynamics \\
WT & Wind tunnel \\
IDDES & Improved Delayed Detached Eddy Simulation \\
C $_{\mathrm{d}}$ & Drag Coefficient \\
MRF & Moving Reference Frame \\
MRFg & Moving Reference Frame - grooves \\
CFL & Courant Friedrichs Lewy \\
CAD & Computer Aided Design \\
S & Slick tyre \\
R & Rain grooved tyre \\
G & Lateral grooved tyre \\
D & Detailed tyre \\
CR & Closed Rim \\
OR & Open Rim \\
\hline
\end{tabular}

